\title{
An Uncommon Cause of Urinary Tract Infection
}

\author{
Kandan Balamurugesan \\ Indira Gandhi Medical College \& RI \\ Kadhiramam, Pondicherry-605009, India \\ pkbala77@hotmail.com \\ Stalin Viswanathan \\ Indira Gandhi Medical College \& RI \\ Kadhiramam, Pondicherry-605009, India \\ stalinviswanathan@ymail.com \\ (Corresponding author)
}

Copyright (C) 2013 Kandan Balamurugesan and Stalin Viswanathan. This is an open access article distributed under the Creative Commons Attribution License, which permits unrestricted use, distribution, and reproduction in any medium, provided the original work is properly cited.

\begin{abstract}
We report a 35 year old man with first episode of urinary tract infection. Clinical examination showed scoliosis and right hand anomalies. Investigations revealed absent kidney on ultrasonogram, mild renal dysfunction and E.coli in urine. Diagnosis of VACTERL association with urinary tract infection was made and he was treated with intravenous ceftriaxone. Renal abnormalities in VACTERL are also reviewed.
\end{abstract}

Keywords: VACTERL; urinary tract infection; renal

\section{Introduction}

Urinary tract infection(UTI) is probably the commonest bacterial infection(Gupta and Trautner 2012). Common risk factors include female sex, pregnancy, diabetes, spinal cord injury, and chronic kidney disease. UTIs are generally infrequent in healthy adult men(Ulleryd 2003). Infections in males are mostly due to prostatic hypertrophy, and structural or functional abnormalities of the urinary tract(Gupta and Trautner 2012). We report a urinary tract infection whose presentation in a 35 year old male led to diagnosis of VACTERL association. 


\section{Case}

This man presented to the Medicine outpatient department with fever, frequency and dysuria of one week duration. On examination he was febrile and had scoliosis, supernumerary thumb on the left and on the right side the thumb, index finger and corresponding carpal bones were absent (Figure 1). He had had no other comorbid conditions. He was born of non-consanguineous parents and there was no family history of similar anomalies. He was married 7 years ago and was detected to have primary infertility. His diagnostic workup in hospital revealed pyuria(15-20 pus cells/hpf), neutrophilic leucocytosis $\left(15 \times 10^{9} / \mathrm{L}\right)$, elevated serum creatinine $(124 \mu \mathrm{mol} / \mathrm{L})$, Escherichia coli in urine culture and an absent kidney on ultrasonogram.. He improved with intravenous ceftriaxone. In view of limb anomalies, scoliosis and renal agenesis, VACTERL association was considered. Imaging revealed scoliosis, extra ribs on both sides and cervical hemivertebrae (Figure1). His echocardiography was normal. There were no trachea-oesophageal fistula or anorectal malformations. He was unwilling for a cystoscopy. He was discharged after one week and was lost to follow up.

\section{Discussion}

VATER (Vertebral anomalies, Anorectal malformations,Tracheo-Esophageal fistula, Renal anomalies) was initially described by Quan and Smith in 1973(Raam, PinedaAlvarez et al. 2011). VACTERL association included cardiac and limb anomalies. Core features like trachea-oesophageal fistula (TEF) and anorectal malformation (ARM) are generally needed for diagnosis three or more are sufficient for diagnosis(Solomon, Raam et al. 2011; Telkes, Reusz et al. 2011). Ten per cent of first degree relatives of VACTERL can have single or multiple features(Solomon 2011). VATER association is seen in an estimated 0.3 to 2.1 of every 10000 live births (Solomon, Raam et al. 2011). Inheritance is sporadic. VACTERL can be associated with chromosomal abnormalities and deletions(Ahn, Mendoza et al. 2009). Some apparent risk factors include maternal diabetes, in-utero estrogen/progesterone exposure like hormonal supplements, statins and infertility treatment(Solomon 2011). Most studies of VACTERL association have revolved around paediatric patients(Raam, Pineda-Alvarez et al. 2011). Presence of ARM and TEF is usually diagnosed in infancy. Cardiac and renal abnormalities may not be discovered until childhood(Raam, Pineda-Alvarez et al. 2011). With better intensive care facilities and surgical options for TEF and ARM there are better chances of survival into adulthood(Raam, Pineda-Alvarez et al. 2011). Also medical complications of primary abnormalities are often seen for the first time in adulthood(Raam, Pineda-Alvarez et al. 2011). 
Vertebral and renal anomalies are generally asymptomatic like in our patient. Vertebral anomalies including those of the ribs are seen in $60-80 \%$ of patients(Solomon 2011). Vertebral anomalies include dysplastic vertebrae, super numerary vertebrae, fusion vertebra(Solomon 2011). Our patient had lumbosacral scoliosis, hemivertebrae and an extra rib on either side. Scoliosis a complication of vertebral anomalies (Solomon 2011) and it was asymptomatic in our patient. Limb abnormalities can be seen 40\%-50\%(Solomon 2011). Radial anomalies are classical although thumb aplasia and polydactyly can be seen(Solomon 2011).

Genitourinary system is the most common organ system involved in VACTERL patients(Kolon, Gray et al. 2000). Fifty to $90 \%$ of patients have renal abnormalities(Solomon 2011; Telkes, Reusz et al. 2011). Renal abnormalities including cystic disease, horseshoe kidney and renal agenesis were seen in $80 \%$ of subjects from a birth defects surveillance registry(Khoury, Cordero et al. 1983). Incidence was 1.6/10000. Genitourinary abnormalities usually occur with hindgut defects due to their intertwined development of the mesoderm(Solomon, Raam et al. 2011). Bladder abnormalities include diverticula and dysplastic bladder and duplication(Ahn, Mendoza et al. 2009). Renal abnormalities are generally associated with ARM although it was not seen in our patient (Solomon 2011). Both hydronephrotic and nonhydronephrotic renal abnormalities occur(Cohen, Kravets et al. 2004). Unilateral agenesis, a non-hydronephrotic abnormality was seen in our patient who was asymptomatic until his infection. Renal agenesis is the most common renal abnormality in VACTERL(Kolon, Gray et al. 2000). Obstructive lesions and bilateral agenesis become symptomatic and are hence ascertained earlier(Khoury, Cordero et al. 1983). Hydronephrosis can occur without obstruction or vesicoureteric reflux(VUR)(Kolon, Gray et al. 2000).

Complications of renal abnormalities in the post-infancy period include VUR, hydronephrosis, nephrolithiasis, urinary tract infection (UTI) and impaired renal function(Solomon 2011). UTI (including pyelonephritis) and nephrolithiasis are generally related to anomalies like ureteral stricture(Raam, Pineda-Alvarez et al. 2011). Upper urinary tract abnormalities also include ureteral ectopia, and obstruction in the ureteropelvic or ureterovesical junction(Kolon, Gray et al. 2000). Urethrocutaneus fistula, rectourethral fistula, stenotic urethra and bulbar urethral stricture are other urinary complications(Ahn, Mendoza et al. 2009). Nephrolithiasis and UTI are the commonest complications in patients with renal anomalies (Raam, Pineda-Alvarez et al. 2011). But complications can occur even without abnormalities(Raam, Pineda-Alvarez et al. 2011). Our patient had renal agenesis and UTI and was not willing for ureterocystoscopy to ascertain lower urinary tract(LUT) abnormalities. But in view of him being asymptomatic until 35 years of age, LUT disorders seem unlikely. Fanconi syndrome, Kallman syndrome and Fraser syndrome are associated with unilateral renal agenesis(Cohen, Kravets et al. 2004). Fanconi's syndrome is the closest differential diagnosis for VACTERL association. The mesoderm of the genitourinary tract and lumbosacral spine are located close together in early embryogenesis and anomalies of both systems can occur 
together(Ahn, Mendoza et al. 2009). This could explain our patient having both vertebral and renal abnormalities without cardiac anomalies, ARM and TEF. Diagnostic modalities in VACTERL are mostly clinical exam for limb anomalies, ARM and TEF, ultra sonogram for renal abnormalities and echocardiography(Solomon 2011). Imaging in male UTIs are usually performed for unresponsive or recurrent infections(Ulleryd 2003). In a study on renal ultrasound and voiding cystourethrography was considered essential as part of initial urological screening in patients with VACTERL(Kolon, Gray et al. 2000).

In conclusion, with better surgical and intensive care facilities and earlier diagnosis, more patients with VACERL association are likely to survive to adulthood. Many patients have undergone renal transplantation and hemodialysis(Ahn, Mendoza et al. 2009). Asymptomatic patients like ours may present for the first time in adulthood. Hence presentation to a physician rather than a paediatrician may ensue. Physicians need to be aware of common infections like UTI occurring in uncommon situations.

\section{References}

1. BD Solomon, MS Raam, DE Pineda-Alvarez, Analysis of genitourinary anomalies in patients with VACTERL (Vertebral anomalies, Anal atresia, Cardiac malformations, Tracheo-Esophageal fistula, Renal anomalies, Limb abnormalities) association, Congenital Anomalies, 51(2011), 87-91.

2. BD Solomon, VACTERL/VATER Association, Orphanet Journal of Rare Diseases, 6(2011), 56.

3. G Telkes, G Reusz, AJ Szabo, RM Langer, A single-center experience with kidney transplantation in the verteberal, anal, cardiac, tracheoesophageal, renal, and limb birth detects (VACTERL) association, Transplant Proceedings, 43(2011), 1250-1251.

4. HL Cohen, F Kravets, W Zucconi, R Ratani, S Shah, D Dougherty, Congenital abnormalities of the genitourinary system, Seminars in Roentgenology, 39(2004), 282-303.

5. K Gupta, B Trautner, In the clinic. Urinary tract infection, Annals of Internal Medicine, 156(2012), ITC1 -16.

6. MJ Khoury, JF Cordero, F Greenberg, LM James, JD Erickson, A population study of the VACTERL association: evidence for its etiologic heterogeneity, Pediatrics, 7(1983), 815-820. 
7. MS Raam, DE Pineda-Alvarez, DW Hadley, BD Solomon, Long-term outcomes of adults with features of VACTERL association, European Journal of Medical Genetics, 54(2011), 34-41.

8. P Ulleryd, Febrile urinary tract infection in men, International Journal of Antimicrobial Agents, 22(2003), 89-93.

9. SY Ahn, S Mendoza, G Kaplan, V Reznik, Chronic kidney disease in the VACTERL association: clinical course and outcome, Pediatric Nephrology, 24(2009), 1047-1053.

10. TF Kolon, CL Gray, RW Sutherland, DR Roth, ET Gonzales, Upper urinary tract manifestations of the VACTERL association,. Journal of Urology, 153 (2000), 1949-1951. 


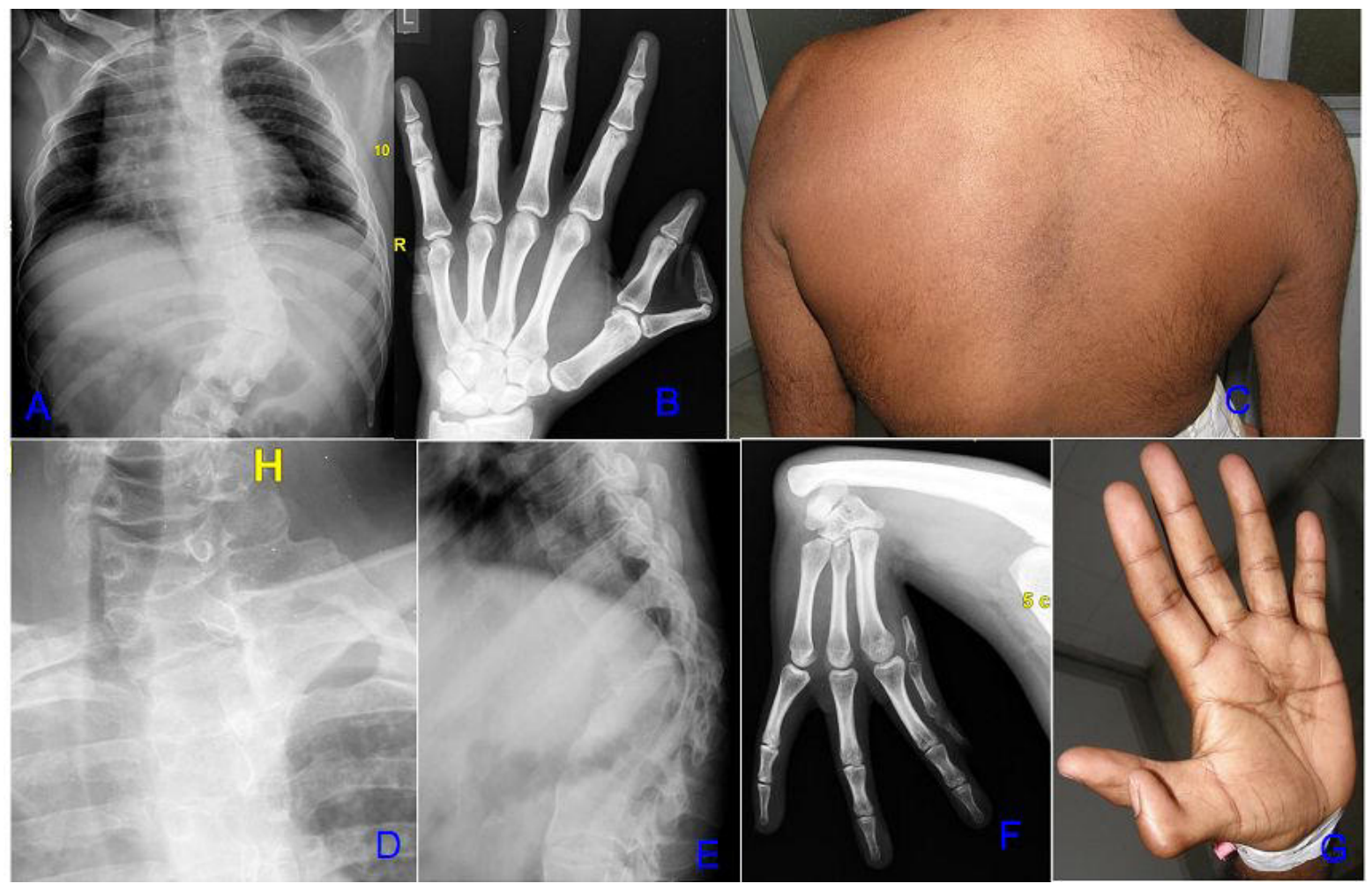

Figure 1A: Plain radiograph of chest and abdomen showing thoracolumbar scoliosis and $13^{\text {th }}$ ribs on both side

Figure 1B: Plain radiograph of right hand shows extra phalanges in the thumb

Figure 1C: Posterior aspect of patient showing scoliosis

Figure 1D: Antero-posterior radiograph of cervical vertebrae revealing hemivertebrae

Figure 1E: Lateral radiograph of thoracolumbar spine, showing kyphosis

Figure 1F: radiograph of forearm and hand revealing absent radius, absent metacarpals ( 1 and 2$)$, absent phalanges of $1^{\text {st }}$ finger and dysplastic phalanges of $2^{\text {nd }}$ finger

Figure 1G: Patient's right hand with supernumerary thumb

Received: November, 2012 